\title{
DCIS
}

https://doi.org/10.29289/259453942021V31S1063

\section{CLINICAL, IMAGING AND THERAPEUTIC CHARACTERISTICS OF 332 PATIENTS WITH DUCTAL CARCINOMA IN SITU (DCIS): A SINGLE- CENTER ANALYSIS}

Marcellus Nascimento Moreira Ramos', Andressa Gonçalves Amorim², Andre Mattar², Luis Henrique Gebrim², Mariana Dinau Leal Passos²

${ }^{1}$ Hospital Pérola Byington, Centro de Referência da Saúde da Mulher - São Paulo (SP), Brazil.

${ }^{2}$ Hospital Pérola Byington-São Paulo (SP), Brazil.

Introduction: Organized mammographic screening led to an increase in the diagnosis of DCIS. In countries with adequate mammographic coverage, the percentage of DCIS reaches $20 \%$. In Brazi, most of the states only perform occasional mammographic screening, and data about DCIS incidence is scarce. Objectives: Analyze and describe clinical, diagnostic, imaging and therapeutic characteristics of patients diagnosed with DCIS and treated at Pérola Byington Hospital (PBH), Brazil. Methods: The official database of PBH from January 2011 to December 2019 showed 11,373 cases of breast cancer, and 812 (7.4\%) of them were DCIS. We analyzed retrospectively the medical record of 332 patients who underwent biopsy, vaccum assisted biopsy guided by mammography or ultrasound whose diagnosis was DCIS, and they were treated at the hospital. Patients with previous breast cancer or lobular neoplasia were excluded. We divided the analysis into two groups, based on the type of surgery they underwent, conservative or radical surgery. Some patients have been submitted to the sentinel lymph node biopsy (SLNB) at surgery and the blue technique stained by H\&E (hematoxylin and eosin) was used. Results: Most patients underwent conservative surgical treatment (73.5\%), with a SLNB in 34.5\%. Mastectomy was performed in $26.5 \%$ of cases and in this group $93.2 \%$ there was also axillary surgery. None of the sentinel nodes was involved. In the conservative surgery group, the mammographic alterations were the main cause for the diagnosis in $80.3 \%$ of the cases, with calcifications being the most common alteration in $73.9 \%$ of the cases, followed by the palpable lesion in $18.5 \%$ of them. Mammography was also the main diagnostic test in the group of patients who underwent mastectomy (73.9\%) and calcifications appeared in $67 \%$ of cases, followed by palpable lesions in $28.1 \%$. When assessing local recurrence, a percentage of $6.4 \%$ was found in conservative surgery (ten in situ and five invasive) and $4.5 \%$ in mastectomy (one in situ and four invasive). Conclusions: The 7.4\% incidence of DCIS shows that even though lower than in countries that have organized screening, it is a growing demand, especially for the public health system where stereotactic biopsy is not available in many centers. Despite the fact that most of the cases were non palpable, we found that mastectomy was indicated in $23.5 \%$ of them, probably because of the extension and multicentricity of the DCIS. The absence of metastases in SNB made us rethink the real need for its indication. 